\title{
APLICACIÓN DE COMPOSITOS FOTO-CATALIZADORES PARA LA DEGRADACIÓN DE P-NITROFENOL EN AGUA
}

\author{
Application of Composites Photocatalysts \\ for The Degradation of $\boldsymbol{P}$-Nitrofenol in Water
}

\author{
${ }^{1}$ Leonardo Medina Nuste, ${ }^{1}$ Marco Bravo Montenegro, ${ }^{1}$ Alex Gavilanes Montoya, \\ ${ }^{1,2}$ Andrés Beltrán Dávalos, ${ }^{1}$ Carlos Medina Serrano*
}

${ }^{1}$ Facultad de Ciencias, Escuela Superior Politécnica de Chimborazo, Riobamba, Ecuador

${ }^{2}$ Escuela Superior Politécnica de Chimborazo, Grupo de Investigación para el Desarrollo Ambiental y Cambio Climático, Riobamba-Ecuador

*carlos.medinas@espoch.edu.ec

La responsabilidad ambiental obliga a buscar vías adecuadas de tratamiento para los desechos líquidos contaminados por compuestos orgánicos y brindar alternativas a las industrias que generan estos efluentes. Por lo cual este trabajo se basa en la degradación de $p$-Nitrofenol (PNF) aplicando compositos de dióxido de titanio $\left(\mathrm{TiO}_{2}\right)$ en carbón activado $\left(\mathrm{CA} / \mathrm{TiO}_{2}\right)$. El fotocatalizador fue soportado sobre carbón en polvo (CA1) y carbón en escamas (CA2), llamados compositos, con el objeto de recuperarlo. Los compositos se caracterizaron con espectrofotómetro IR y microscopio de barrido electrónico. La degradación del contaminante fue evaluada con espectrofotómetro UV-visible. Para los ensayos con luz UV se utilizó una lámpara $(\lambda=365 \mathrm{~nm})$ para irradiar el reactor durante todo el proceso y $\mathrm{H}_{2} \mathrm{O}_{2}$ como agente oxidante. Una vez estudiados los distintos ensayos usando un diseño experimental completamente aleatorio, se observó que la combinación de CA1 muestra una alta eficiencia $(99,1 \%)$ de remoción de PNF en una solución acuosa concentrada (250mg L-1), en un tiempo medio de 80 minutos, pues CA2 presenta una remoción menor $(50,46 \%)$ en 80 minutos de tratamiento. Con esto se puede concluir que CA1 presenta un mejor acomplejamiento y eficiencia en la degradación de PNF.

Palabras claves: $p$-Nitrofenol, degradación fotocatalítica, carbón activado, dióxido de titanio, composito.

\section{A bstract}

The Environmental responsibility forces us to look for adequate ways to treat contaminated wastewater by organic compounds and provide alternatives to the generator industries. Therefore, this work is based on the degradation of p-Nitrophenol (PNF) applying $\mathrm{TiO}_{2}$ composites on activated carbon $\left(\mathrm{CA} / \mathrm{TiO}_{2}\right)$. The photocatalyst was supported on carbon powder (CA1) and carbon in flakes (CA2) which are called composites in order to recover it. The compounds were characterized with IR spectrum and electronic scanning microscope. The degradation of the pollutant was evaluated with a UV-visible spectrophotometer. A UV light $(\lambda=365 \mathrm{~nm})$ was used to irradiate the reactor and $\mathrm{H} 2 \mathrm{O} 2$ was used as an oxidizing agent throughout the whole process time. Once the different trials have been studied using a completely randomized experimental design, it was observed that the combina- 
tion of CA1 shows a high efficiency (99.1\%) of removal of PNF in a concentrated aqueous solution (250mg.L-1) in an average time of 80 minutes. Thus, CA2 presents a minor removal $(50.46 \%)$ in an 80 minutes treatment. It might be conclude that CA1 presents a better complexation and efficiency in the PNF degradation.

Keywords: p-Nitrophenol; photocatalytic degradation; Activated carbon; titanium dioxide; composite

\section{INTRODUCCIÓN}

Los nitrofenoles en el agua, constituyen una problemática ambiental causada principalmente por las descargas líquidas de la industria textil, farmacéutica e industria de hierro y acero, como fuentes puntuales y difusas por la degradación de plaguicidas como el paratión y el fluoridifen (1) (2). El compromiso por cuidar el ambiente nos llevar a usar alternativas eficientes tales como los procesos de oxidación avanzada (POAs) para el tratamiento de efluentes con contaminantes emergentes (3). Los tratamientos fotocatalíticos han mostrado ser muy eficientes para degradar diferentes compuestos orgánicos altamente peligrosos (4) (5) (6) (2).

Los POAs son técnicas que utilizan fuentes de luz natural, y/o artificial para irradiar y desencadenar las reacciones degradativas radicalarias, como el radical hidroxilo que mineraliza los contaminantes (7), siendo frecuente en los compuestos orgánicos derivados del benceno (8-10). El $\mathrm{TiO}_{2}$ es apropiado y de uso común en los POAs, debido a sus características como: alta fotoactividad, inercia química, no toxicidad y bajo costo $(11,12)$ que actúa junto al reactivo foto-fenton como semiconductores de la luz activando la mineralización de los contaminantes, (13).

El dióxido de Titanio así como otros semiconductores se puede soportar sobre materiales como: la ciclodextrina (13) y carbón activado, siempre que no se elimine su actividad catalizadora (14) y siendo el más eficiente el carbón activado modificado mediante calentamiento con ácido nítrico-urea- $\mathrm{N}_{2}$ a $800{ }^{\circ} \mathrm{C}$ (12). La finalidad de usar estos materiales que inmovilizan el catalizador es recuperar por gravedad el semiconductor que debido a su tamaño nano-métrico requiere procesos industriales de centrifugación para removerlo del seno del efluente tratado, siendo el limitante para su aplicación industrial junto con la manufactura y regeneración del carbón activado $(8,15)$.

Los compuestos nitroaromáticos, son contaminantes altamente tóxicos que se resisten a tratamientos químicos comunes $(1,16)$. La Agencia de Protección Medioambiental de los Estados Unidos (USEPA) considera al PNF como uno de los once compuestos contaminantes fenólicos prioritarios. La Comunidad Europea establece, como concentración máxima admisible de fenoles en agua de consumo humano, $0.1 \mu \mathrm{g}$.L-1 en contenido individual y $0.5 \mu \mathrm{g} . \mathrm{L}-1$ en contenido total (17). El interés de este estudio, es evaluar la degradación de PNF como contaminante de aguas residuales, aplicando procesos combinados de oxidación avanzada tales como TiO2/H2O2/luz UV, donde el $\mathrm{TiO} 2$ se encuentra soportado sobre dos tipos de carbón activado (composito) en escamas y en polvo, con la finalidad de recuperar fácilmente el TiO2 luego de tratamiento foto-degradativo.

\section{MATERIAL Y MÉTODOS}

\section{Síntesis de los compositos de $\mathrm{TiO}_{2} / \mathrm{CA}$}

titanio del polimorfo de cristal de anastasa fue utilizado para la síntesis de cada uno de los compositos, por tener mejor actividad catalítica en comparación con sus otros polimorfos de cristales de $\mathrm{TiO}_{2}$ (rutilo y brookita) $(18,14)$. La acomplejación fue realizada de forma directa sin ayuda de ningún precursor, sometiendo a tratamiento térmico a $400{ }^{\circ} \mathrm{C}$ como mecanismo de síntesis. Los compositos se obuvieron mezclando $\mathrm{TiO}_{2}$ con carbón en pol- 
vo (CA1) y carbón en escamas (CA2) y sometidos a un pre-tratamiento para mejorar la actividad catalítica con $500 \mathrm{ml} \mathrm{de} \mathrm{H}_{2} \mathrm{O}_{2}(110 \mathrm{~V})$ por 18 horas, y secado a $105^{\circ} \mathrm{C}$ en una estufa CONTERM por 24 horas (20). Así mismo se sintetizaron CA1 y CA2 sin pre-tratamiento con la finalidad de diferenciar los porcentajes de $\mathrm{TiO}_{2}$ adheridos a las paredes del soporte.

La síntesis de los compositos se realizó mezclando y agitando $\mathrm{TiO}_{2}$ a una relación de 1 y $5 \%$ (100 y $\left.160 \mathrm{~g}\right)$, frente al CA, añadido a los diez minutos posteriores, a la suspensión formada con el agua destilada en proporción: de $5 \mathrm{ml} / \mathrm{g}$ de CA. La suspensión se calentó a temperatura de ebullición del agua hasta conseguir una fase semisólida, la cual se llevó a la estufa de secado por 24 horas a $105^{\circ} \mathrm{C}$, después se la calcinó a $450{ }^{\circ} \mathrm{C}$ por 2 horas en una mufla SELECT-HORN-TFT, para activar la fase anatasa del $\mathrm{TiO}_{2}$ adherido al soporte. Una vez frío el composito se lavó con agua destilada para eliminar el excedente de $\mathrm{TiO}_{2}$ no adherido al soporte y secado a $105^{\circ} \mathrm{C}$ por 24 horas en la estufa (21).

\section{Caracterización fisicoquímica de cada composito de $\mathrm{TiO}_{2} / \mathrm{CA}$ sintetizado}

Los compositos fueron analizados en Microscopio Electrónico de Barrido (Jeol Modelo JSM-IT 100). Se realizó un total de 4 análisis de espectrofotometría de dispersión de RX (EDS), con el objetivo de determinar la diferencia de adhesión del $\mathrm{TiO}_{2}$ en CA1 y CA2 tanto con pretratamiento con sin él. Para las lecturas, las muestras fueron colocadas sobre el porta muestras y fijadas con Copper Tape (cinta conductora que facilita la señal de respuesta del equipo).

De la misma manera, se tomaron las imágenes de la superficie de cada uno de los compositos, para poder comparar su porosidad, forma y estructura. Pues depende de la porosidad y superficie de contacto de CA1 y CA2, la adhesión del $\mathrm{TiO}_{2}$ sobre las paredes de los mismos.

\section{Evaluación de la actividad foto-catalítica.}

Para evaluar los compositos sintetizados como fotocatalizadores, se realizaron pruebas de degradación de fotocatálisis heterogénea en soluciones de PNF preparadas en laboratorio a $250 \mathrm{mg}$.L-1 de concentración inicial. A través de un diseño experimental completamente aleatorio (DCA) se plantean 44 posibles experimentos combinando tres factores (diferentes compositos, agitación y catalizador) de los cuales se seleccionaron 9 experimen- tos realizados por triplicado e irradiados todo el tiempo con una fuente de luz UV. La luz UV empleada fue una lámpara de $9 \mathrm{~W}$, la cual se ubicó en la parte superior de un recipiente de 1 litro a una distancia aproximada de $1 \mathrm{~cm}$ de la superficie de la solución. El volumen de solución tratada por ensayo fue de 600 $\mathrm{ml}$ y la relación de composito empleado fue $2,5 \mathrm{~g}$ composito por litro solución. Las soluciones fueron tratadas durante 80 minutos. Se realizaron pruebas con: $\mathrm{TiO}_{2}$ y carbón activado virgen (escamas y polvo) para evaluar el comportamiento del composito en la oxidación del PNF frente a la acción individual de sus componentes.

\section{Proceso analítico}

Iniciada la fase degradativa, se tomó alícuotas de $9 \mathrm{ml}$ directamente del reactor en periodos de $5,10,20,40$ y $80 \mathrm{mi}$ nutos. Las muestras son centrifugadas en una Centrífuga Hermle (Z200A) a 6000 rpm durante 2 minutos y colocadas inmediatamente en una cámara oscura para evitar el contacto con la luz natural interrumpiendo el proceso foto-oxidativo hasta su respectivo análisis en el Espectrofotómetro UV-Visible (Thermo BioMate 3S) a $400 \mathrm{~nm}$. Para cuantificar la concentración de PNF en las muestras tomadas se usó la recta de calibrado, que relaciona la absorción de una muestra con una concentración conocida de PNF según la ley de Lambert-Beer.

\section{RESULTADOS Y DISCUSIÓN}

\section{Síntesis y caracterización de los com- positos de $\mathrm{TiO}_{2} /$ carbón activado}

La composición de los ocho compositos foto-catalizadores está representada en la tabla 1, que indica la mezcla de los soportes de carbón activado en polvo (CA1) y carbón activado en escamas (CA2) con y sin pretratamiento. 


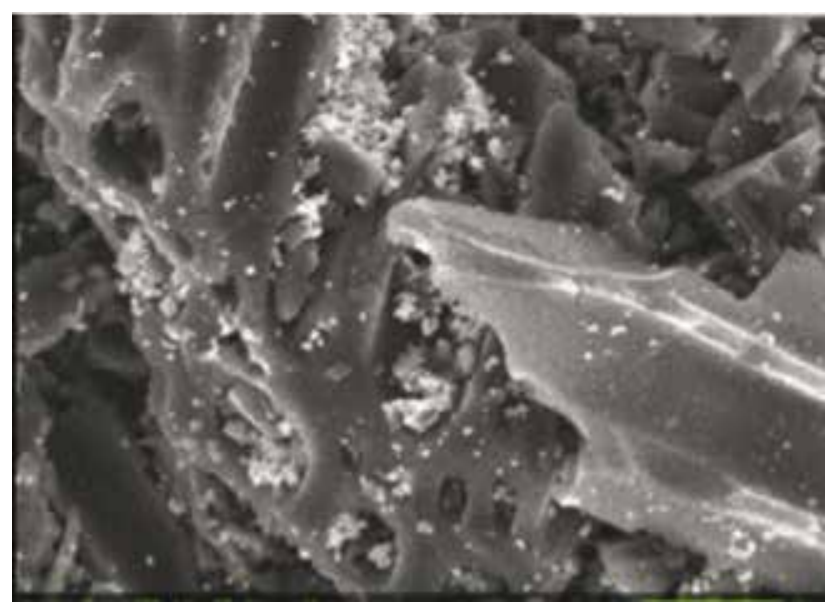

a. Composito en polvo (CA1)

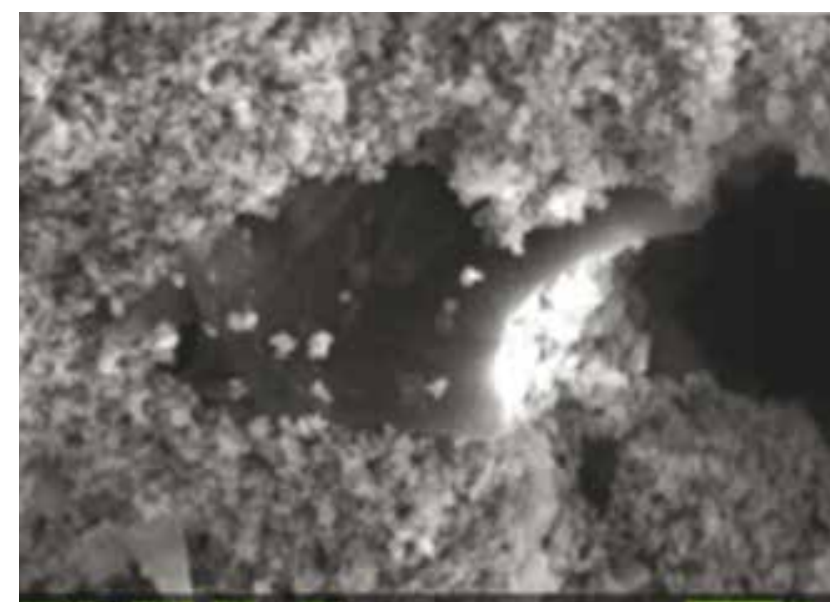

b. Composito en escamas (CA2)

Figura 1. Dióxido de titanio al $5 \%$ soportado en carbón activado en polvo y escamas

Las pruebas de caracterización cualitativa mostradas por el microscopio electrónico de barrido (SEM), permitieron validar la adherencia del catalizador a las paredes del carbón activado en polvo y en escama (Fig.1).

No obstante, los análisis de EDS indican que existe una menor concentración de Ti en los compositos sometidos a pretratamiento oxidativo. Por lo tanto se verifica que el peróxido de hidrógeno $\left(\mathrm{H}_{2} \mathrm{O}_{2}\right)$ no ayuda a mejorar el proceso de

\begin{tabular}{|l|c|c|c|c|}
\hline Compositos & $\mathbf{T i O}_{\mathbf{2}}(\mathbf{g})$ & $\mathbf{C A}(\mathbf{g})$ & $\mathbf{H}_{\mathbf{2}} \mathbf{O}_{\mathbf{2}}(\mathbf{m L})$ & Nomenclatura \\
\hline Composito 1 & 8 & 160 & 0 & CAESP5\% \\
\hline Composito 2 & 1,6 & 160 & 0 & CAESP1\% \\
\hline Composito 3 & 5 & 160 & 0 & CAPSP5\% \\
\hline Composito 4 & 1 & 160 & 0 & CAOSP1\% \\
\hline Composito 5 & 8 & 160 & 800 & CAECP5\% \\
\hline Composito 6 & 1,6 & 160 & 800 & CAECP1\% \\
\hline Composito 7 & 5 & 100 & 500 & CAPCP5\% \\
\hline Composito 8 & 1 & 100 & 500 & CAPCP $1 \%$ \\
\hline
\end{tabular}

Tabla 1. Composición porcentual de los compositos sintetizados
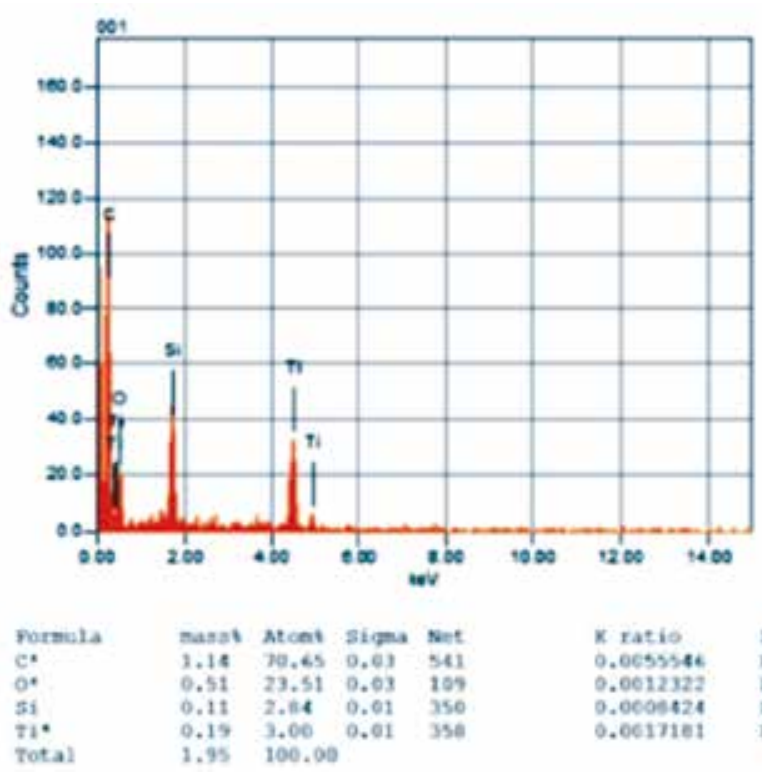

a. Composito en polvo (CA1)
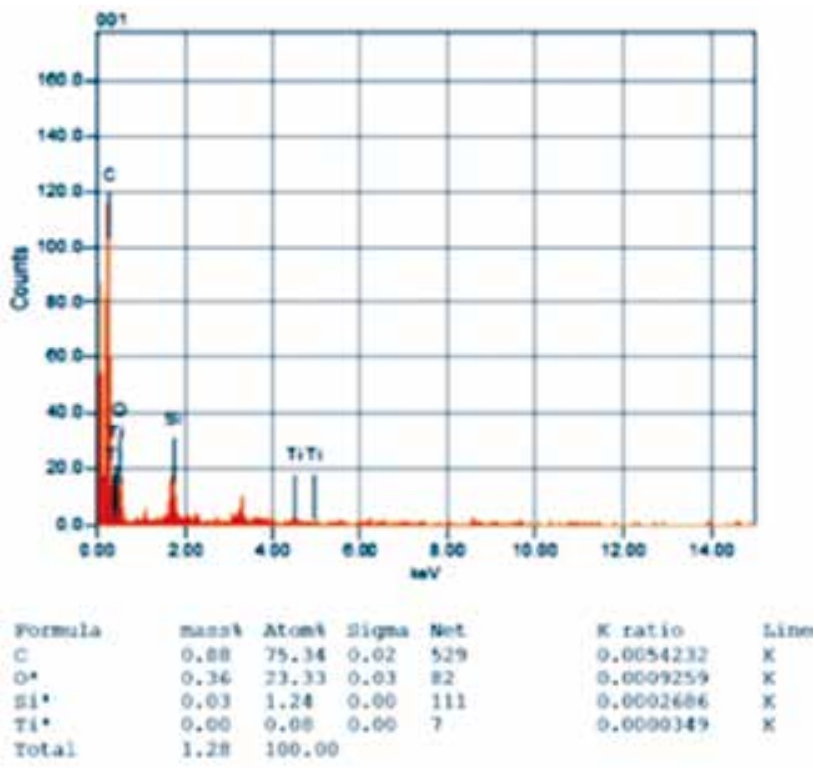

b. Composito en escamas (CA2)

Figura 2. Análisis EDS de los compositos 


\begin{tabular}{|c|c|c|c|c|c|c|}
\hline \multirow{2}{*}{ Tiempo (min) } & \multicolumn{6}{|c|}{ Concentración (mg. $\left.\mathrm{L}^{-1}\right)$} \\
\hline & $\mathbf{0}$ & 5 & 10 & 20 & 40 & 80 \\
\hline CAPSP5\% $\%^{a}$ & 256,90 & 27,65 & 21,53 & 18,47 & 16,28 & 8,81 \\
\hline CAPSP5\% ${ }^{b}$ & 256,90 & 30,01 & 22,49 & 21,22 & 20,46 & 10,92 \\
\hline CAESP1\% ${ }^{c}$ & 256,90 & 197,29 & 190,61 & 184,43 & 179,36 & 162,63 \\
\hline CAESP5\% ${ }^{d}$ & 256,90 & 222,28 & 211,86 & 208,70 & 202,99 & 200,29 \\
\hline САЕСР5\% ${ }^{\mathrm{e}}$ & 256,90 & 174,61 & 165,76 & 155,60 & 143,77 & 127,27 \\
\hline C(A) POLVO ${ }^{1}$ & 256,90 & 11,88 & 8,53 & 6,99 & 4,42 & 2,72 \\
\hline C(A) ESCAMAS ${ }^{2}$ & 256,90 & 245,60 & 238,65 & 233,41 & 229,58 & 223,00 \\
\hline C(A) ESCAMAS ${ }^{3}$ & 256,90 & 252,20 & 245,33 & 242,95 & 234,12 & 168,22 \\
\hline $\mathrm{TiO}_{2}{ }^{4}$ & 256,90 & 220,26 & 206,80 & 199,87 & 192,92 & 182,63 \\
\hline
\end{tabular}

${ }^{\mathrm{a}}$ Con Agitación - Con Catalizador; ${ }^{\mathrm{b}}$ Con Agitación-Sin Catalizador; ${ }^{\mathrm{c}}$ Sin Agitación-Con Catalizador; ${ }^{\mathrm{d}}$ Sin Agitación-Sin Catalizador; ${ }^{\mathrm{e}}$ Sin Agitación-Con Catalizador. ${ }^{1}$ Sin Agitación-Con Catalizador; ${ }^{2}$ Con Agitación - Con Catalizador; ${ }^{3}$ Con Agitación-Sin Catalizador; ${ }^{4}$ Sin Agitación-con Catalizador

Tabla 2. Degradación fotocatalítica de PNF usando compositos en función del tiempo

adherencia del $\mathrm{TiO}_{2}$ en el carbón activado como lo recomiendan trabajos previos (21).

\section{Actividad foto-catalítica de cada composito en la degradación de PNF}

De acuerdo a la figura 3, el comportamiento degradativo del composito CAPSP5\% y CAPSP5\% es similar a la tendencia del carbón activado en polvo virgen, pero la diferencia radica en que el carbón en polvo no degrada, sino que elimina de la solución el PNF adherido en sus paredes. Así mismo CAPSP5\%a y CAPSP5\%b indica que la adición del agente oxidante no es significativa en la degradación con carbón activado en polvo. Por otro lado, los compositos con carbón en escamas CAESP5\%d y CAESP5\%e, muestran una disminución mucho menor de la concentración del contaminante en el tiempo establecido.

Para todos los casos, en los primeros 5 minutos de tratamiento se consiguió la mayor parte de remoción del contaminante, mientras que una vez alcanzado el equilibrio se mantiene una tendencia de degradación constante. En el caso del carbón activado virgen la disminución de la concentración del PNF que se debe al material adsorbente, que retiene

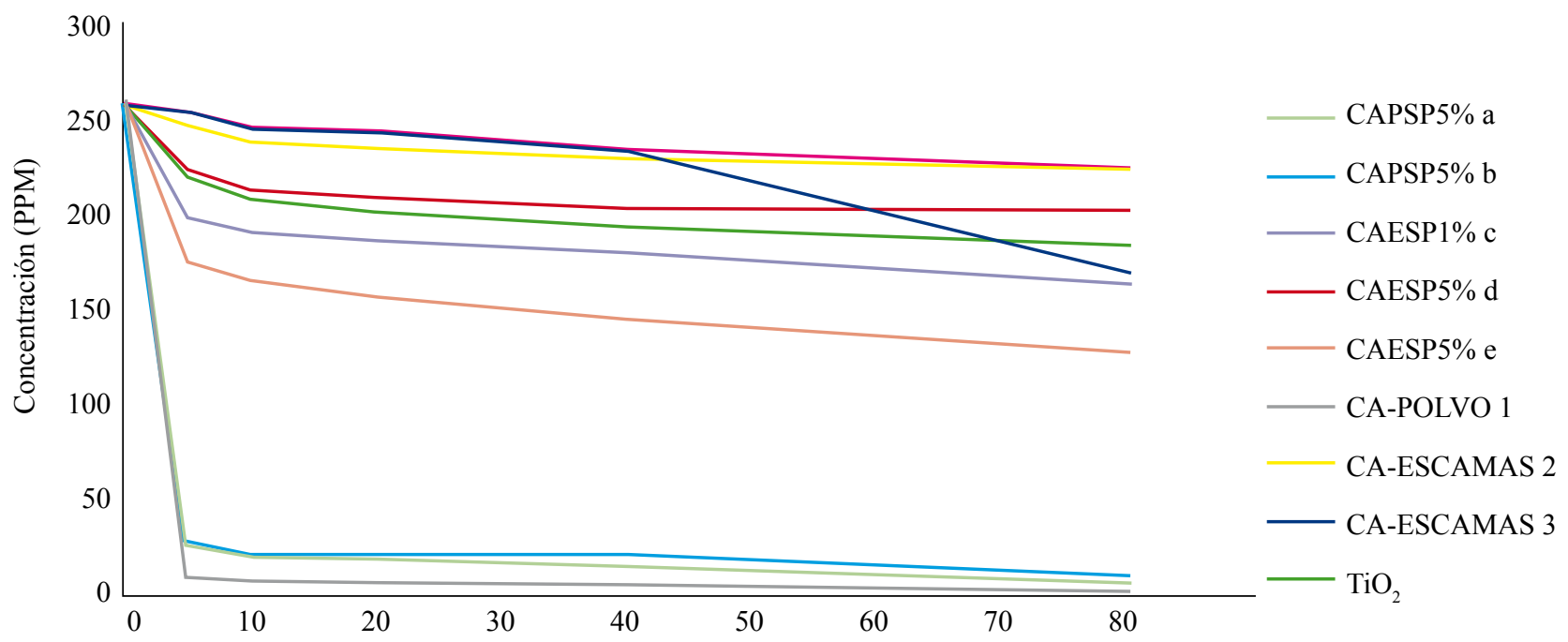

Figura 3. Cinética de degradación del PNF 


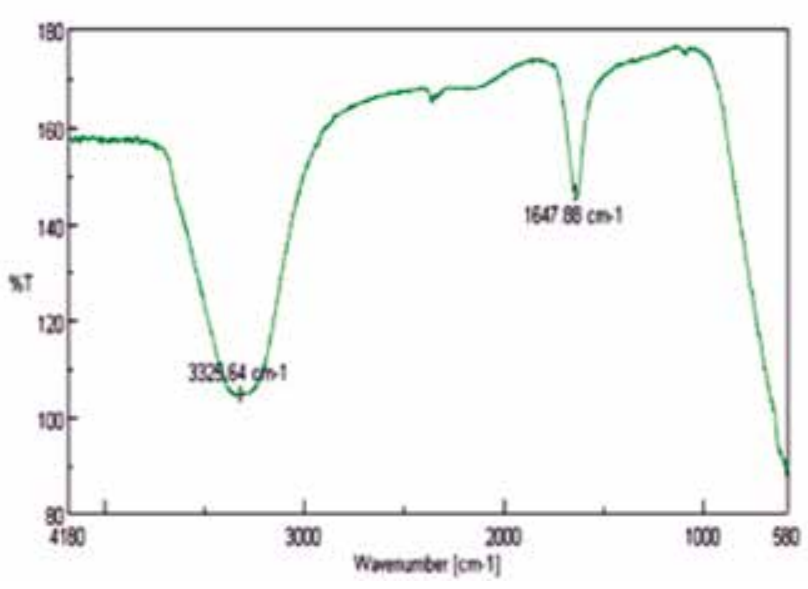

a. Espectro IR de la Solución Inicial de $p$-Nitrofenol

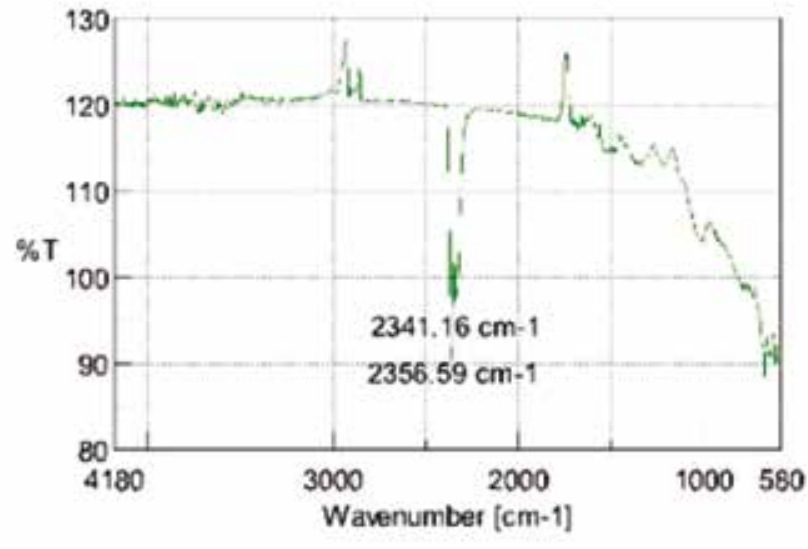

b. Espectro IR de carbón activado en polvo virgen

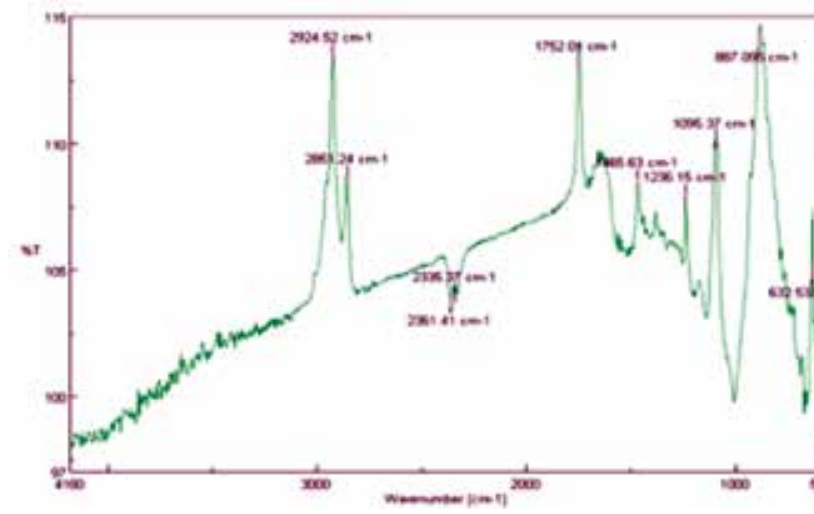

c. Espectro IR del composito de carbón activo en polvo post tratamiento

Figura 4. Espectros Infra Rojo de los compositos analizados post tratamiento fotocatalíticos

en su superficie cierta cantidad inicial de PNF hasta saturar sus poros, y no necesariamente hay una degradación fotocatalítica.

La Figura 3 muestra la comparación de las capacidades de remoción de PNF para cada uno de los compositos.

Con la intensión de verificar si el composito únicamente adsorbe el contaminante o lo degrada, se hicieron pruebas en el Espectrofotómetro de Infrarrojo, determinando los grupos funcionales presentes en la fase sólida luego del tratamiento En la Fig. 4, se muestra los picos característicos de la solución de PNF, los cuales son comparados con los análisis del carbón activado virgen y composito postratamiento.
El composito de carbón en polvo analizado luego del tratamiento fotocatalítico no presenta ningún pico similar al de la solución inicial de PNF (figura 4 a), lo que deja evidencia de que efectivamente el composito ha degradado el contaminante y mineralizado en sus respectivos productos de descomposición. Los espectros IR de carbón virgen y del composito de carbón activo en polvo postratamiento indican que el carbón activado por su capacidad absorbente retiene el contaminante, condicionado por la posición del grupo $-\mathrm{NO}_{2}$ en el anillo de benceno(22); y la acción catalítica del $\mathrm{TiO}_{2}$ adherido a las paredes del soporte y la acción de la luz UV, degradan en su interior el contaminante.

Los resultados del estudio se presentan de acuerdo a 6 factores: agitación, adición de catalizador, tiempo, porcentaje de impregnación de $\mathrm{TiO} 2$, tipo de carbón activado y pre-tratamiento oxidativo al soporte. Estos factores influyen en la eficiencia de los diferentes ensayos de 
degradación. Para cada tipo de composito se realizó las pruebas de degradación a una misma concentración de $\mathrm{PNF}$, donde se comprobó que el CA en polvo, tiene un efecto absorbente muy efectivo en la remoción del PNF y que luego de analizar el composito post tratamiento, el PNF no solo fue retenido sino degradado.

Analizando los resultados de la Figura 3, se aprecia que efectivamente los porcentajes de remoción del PNF de la solución acuosa, presentan una eficiencia en al menos un 99,6\% para el caso de los compositos foto-catalizadores, el carbón en polvo virgen presenta un porcentaje de remoción de 99,89\%. El composito más óptimo en la degradación del PNF, es CAPSP5\% que mantienen mayor adherencia al soporte, al existir más partículas del catalizador consiguiendo la degradación del contaminante en un menor tiempo.

El análisis de degradación del PNF (tabla 2) indica que los valores más eficientes son aquellos cuyo soporte es el carbón activado en polvo, esto debido, a que el carbón activado en polvo posee una gran superficie de contacto en sus poros capaces de retener suficiente catalizador para que este luego interactúe con el contaminante y con ayuda de la luz UV, mineralice el PNF.

\section{CONCLUSIONES}

El composito fotocatalizador CA1, sintetizado para la degradación de PNF (250mg.L-1) fue el resultado de la mezcla fisico-química de carbón activado en polvo con catalizador (dióxido de titanio) al 5\% (porcentaje que se adhiere mayoritariamente al carbón activado), con agitación y agente oxidante. Todos estos factores combinados presentan una efectividad del 96,5\% de remoción del contaminante PNF en 80 minutos. Alcanzando el equilibrio de degradación dentro de los primeros 5 minutos, en los cuales se logra mineralizar la mayor parte del contaminante, luego del cual la velocidad de reacción no varía significativamente en los 80 minutos de tratamiento. Por su parte el composito CA2 presentó una remoción máxima del $50.5 \%$ del PNF en el mismo tiempo de tratamiento. En el estudio de degradación utilizando carbón activado puro se elimina el 98,9\% del PNF, pero este solo se elimina por adsorción en los poros del carbón activado hasta saturación, más no degradación del contaminate. El dióxido de titanio puro por su parte elimina el $28,9 \%$ mineralizando el PNF.

\section{eferencias}

1. Borras C, Berzoy C, Mostany J, Scharifker BR. Efecto de la concentración inicial y el potencial de electrodo sobre la cinética de oxidación y la eficiencia de mineralización del p-nitrofenol sobre electrodos de sb-sno 2. 2006;xviii(1).

2. Foo KY, Hameed BH. Decontamination of textile wastewater via TiO2/activated carbon composite materials. Adv Colloid Interface Sci [Internet]. 2010;159(2):130-43. Available from: http://dx.doi. org/10.1016/j.cis.2010.06.002

3. Rodriguez-Narvaez OM, Peralta-Hernandez JM, Goonetilleke A, Bandala ER. Treatment technologies for emerging contaminants in water: A review. Chemical Engineering Journal. 2017.

4. Shaoqing Y, Jun H, Jianlong W. Radiation-induced catalytic degradation of p-nitrophenol (PNP) in the presence of TiO2nanoparticles. Radiat Phys Chem [Internet]. 2010;79(10):1039-46. Available from: http://dx.doi.org/10.1016/j.radphyschem.2010.05.008

5. Rodrigues CSD, Soares OSGP, Pinho MT, Pereira MFR, Madeira LM. p-Nitrophenol degradation by heterogeneous Fenton's oxidation over activated carbon-based catalysts. Appl Catal B Environ [Internet]. 2017;219:109-22. Available from: http://dx.doi.org/10.1016/j.apcatb.2017.07.045

6. Selishchev DS, Kolinko PA, Kozlov D V. Influence of adsorption on the photocatalytic properties of $\mathrm{TiO} 2 / \mathrm{AC}$ composite materials in the acetone and cyclohexane vapor photooxidation reactions. J Photochem Photobiol A Chem [Internet]. 2012;229(1):11-9. Available from: http://dx.doi. org/10.1016/j.jphotochem.2011.12.006 
7. Barrera-Díaz C, Cañizares P, Fernández FJ, Natividad R, Rodrigo MA. Electrochemical Advanced Oxidation Processes: An Overview of the Current Applications to Actual Industrial Effluents. Chem Soc J Mex Chem Soc. 2014;

8. Byrne C, Subramanian G, Pillai SC. Recent advances in photocatalysis for environmental applications. J Environ Chem Eng [Internet]. 2017;(July):0-1. Available from: http://dx.doi.org/10.1016/j. jece.2017.07.080

9. Martins AC, Cazetta AL, Pezoti O, Souza JRB, Zhang T, Pilau EJ, et al. Sol-gel synthesis of new $\mathrm{TiO} 2 /$ activated carbon photocatalyst and its application for degradation of tetracycline. Ceram Int [Internet]. 2017;43(5):4411-8. Available from: http://dx.doi.org/10.1016/j.ceramint.2016.12.088

10. Ouzzine M, Romero-Anaya AJ, Lillo-Ródenas MA, Linares-Solano A. Spherical activated carbon as an enhanced support for TiO2/AC photocatalysts. Carbon N Y. 2014;67:104-18.

11. Asiltürk M, Şener Ş. TiO 2-activated carbon photocatalysts: Preparation, characterization and photocatalytic activities. Chem Eng J. 2012;180:354-63.

12. Brienza M, Katsoyiannis IA. Sulfate radical technologies as tertiary treatment for the removal of emerging contaminants from wastewater. Sustain. 2017;

13. Liang C, Liu Y, Li K, Wen J, Xing S, Ma Z, et al. Heterogeneous photo-Fenton degradation of organic pollutants with amorphous Fe-Zn-oxide/hydrochar under visible light irradiation. Sep Purif Technol [Internet]. 2017;188:105-11. Available from: http://dx.doi.org/10.1016/j.seppur.2017.07.027

14. Zhang J, Huang ZH, Xu Y, Kang FY. Carbon-coated TiO2composites for the photocatalytic degradation of low concentration benzene. Xinxing Tan Cailiao/New Carbon Mater [Internet]. 2011;26(1):63-70. Available from: http://dx.doi.org/10.1016/S1872-5805(11)60067-X

15. Koyuncu H, Yildiz N, Salgin U, Köroĝlu F, Çalimli A. Adsorption of o-, m- and p-nitrophenols onto organically modified bentonites. J Hazard Mater. 2011;

16. Zhang CL, Yu YY, Fang Z, Naraginti S, Zhang Y, Yong YC. Recent advances in nitroaromatic pollutants bioreduction by electroactive bacteria. Process Biochem [Internet]. 2018;(March):0-1. Available from: https://doi.org/10.1016/j.procbio.2018.04.019

17. Silva Vargas ME. Preconcentracion selectiva de fenoles contaminantes prioritarios y determinación por espectrofotometría uv-visible, cromatografía líquida de alta eficacia y cromatografía de gases/espectrometría de masas. universidad complutense de madrid; 2000.

18. Awfa D, Ateia M, Fujii M, Johnson MS, Yoshimura C. Photodegradation of pharmaceuticals and personal care products in water treatment using carbonaceous-TiO 2 composites: A critical review of recent literature. Water Res. 2018;

19. Zhang J, Zhou P, Liu J, Yu J. New understanding of the difference of photocatalytic activity among anatase, rutile and brookite TiO2. Phys Chem Chem Phys. 2014;

20. Devi LG, Anitha BG. Exploration of vectorial charge transfer mechanism in $\mathrm{TiO} 2 / \mathrm{SrTiO} 3 \mathrm{com}-$ posite under UV light illumination for the degradation of 4-Nitrophenol: A comparative study with TiO2and SrTiO3. Surfaces and Interfaces. 2018;

21. Murillo HA. Obtención de un composito de dioxido de titanio y carbon activado a la oxidación fotocatalítica del ión cianuro. 2015;205. Available from: https:/www.google.com.ec/ search?q=Extracción + del + titanil + sulfato $+d e l+$ mineral + ilmenita $+y+$ su+evaluación+en + la $+e s-$ terificación + del+ácido+oléico.\&oq=Extracción + del + titanil + sulfato + del + mineral + ilmenita $+y+-$ su+evaluación+en+la+esterificación+del+ácido+oléico.\&aqs

22. Altaher H, Dietrich AM. Characterizing o- and p-nitrophenols adsorption onto innovative activated carbon prepared from date pits. Water Sci Technol. 2014; 